\title{
Avaliação de alguns parâmetros ecocardiográficos do gato-do-mato (Leopardus tigrinus), mantido em cativeiro e submetido à anestesia com xilazina e quetamina
}

\author{
[Evaluation of some echocardiographic parameters of Oncilla (L. tigrinus), kept in captivity and submitted \\ to anesthesia with xilazine and ketamine] \\ P.S.L. Carvalho ${ }^{1}$, G.G. Pereira ${ }^{1}$, L.C.Petrus ${ }^{1}$, E.C. Soares ${ }^{2}$, \\ L.E. Michima ${ }^{2}$, M.H.M.A. Larsson ${ }^{3 *}$ \\ ${ }^{1}$ Médico veterinário autônomo \\ ${ }^{2}$ Aluno de pós-graduação - FMVZ-USP - São Paulo, SP \\ ${ }^{3}$ Faculdade de Medicina Veterinária e Zootecnia - USP \\ Av. Prof. Orlando Marques de Paiva, 87 \\ 05508-900 - São Paulo, SP
}

\begin{abstract}
RESUMO
Avaliaram-se alguns parâmetros ecocardiográficos em modos B, M e Doppler de 27 gatos-do-mato, Leopardus tigrinus, pequeno felídeo selvagem, mantidos em cativeiro e submetidos à anestesia com 1 a $2 \mathrm{mg} / \mathrm{kg}$ de xilazina e $10 \mathrm{mg} / \mathrm{kg}$ de quetamina. Observaram-se alterações dos parâmetros cardiovasculares quando os resultados foram comparados aos do gato doméstico (Felis catus) não anestesiado.
\end{abstract}

Palavras-chave: gato-do-mato, Leopardus tigrinus, Felis catus, parâmetros ecocardiográficos, quetamina, xilazina

\section{ABSTRACT}

Some echocardiographic parameters in B, M-mode and Doppler of 27 Oncillas, Leopardus tigrinus, a wild little feline, kept in captivity and submitted to anesthesia with 1 to $2 \mathrm{mg} / \mathrm{kg}$ of xilazine and $10 \mathrm{mg} / \mathrm{kg}$ of ketamine, had been evaluated. Changes of the cardiovascular parameters were observed when the results were compared to non anesthetized domestic cat (Felis catus).

Keywords: Leopardus tigrinus, Felis catus, echocardiography parameters, ketamine, xilazine

\section{INTRODUÇ̃̃O}

Os pequenos felídeos selvagens lembram, em vários aspectos, o gato doméstico (Felis catus) por terem porte e peso reduzidos, e muitos, por sua aparência e pelagem.

O gato-do-mato (Leopardus tigrinus), também chamado de gato-do-mato-pequeno ou pintadinho, é uma das menores espécies de felinos presentes no território nacional. Com proporções bem semelhantes às do gato doméstico, pesa entre 1,75 e $3,5 \mathrm{~kg}$, com média de $2,3 \mathrm{~kg}$, e seu comprimento corpóreo é de cerca de $49 \mathrm{~cm}$, sendo o macho maior que a fêmea. Esse animal tem as patas pequenas e delicadas e a cauda muito longa. Sua pelagem apresenta coloração variável entre os indivíduos, com tonalidades entre amarelo claro e castanho amarelado, com manchas pequenas e escuras espalhadas pelo corpo. Esse pequeno felino pode ser encontrado desde a Costa Rica até a Argentina e em todo o Brasil.

A cardiologia tem tido grandes avanços na área de diagnóstico, sendo o maior deles a possibilidade de se realizar um exame do coração por meio não invasivo, com o uso do

Recebido em 13 de fevereiro de 2007

Aceito em 30 de abril de 2007

* Autor para correspondência (corresponding author)

E-mail: akaolar@usp.br 
ecocardiograma (Allen e Downey, 1983; Yamato, 2001).

Em felinos, a ecocardiografia é utilizada com muita freqüência para diagnosticar as cardiopatias, em especial as alterações do miocárdio, caracterizando e diferenciando o tipo de cardiomiopatia (CM) (Moise et al., 1986; Bonagura, 2000). Em função da ecocardiografia, houve um aumento significativo do conhecimento das doenças miocárdicas, elevando e melhorando os diagnósticos realizados.

O melhor meio de diagnóstico definitivo e diferencial entre as cardiomiopatias é o ecocardiograma (Bonagura, 2000; Schwartz, 2003), mostrando a grande importância da ecocardiografia na clínica de felinos.

A finalidade deste estudo foi avaliar alguns parâmetros ecocardiográficos, bem como observar a ocorrência ou não de distúrbios miocárdicos em gatos-do-mato (Leopardus tigrinus), mantidos em cativeiro.

\section{MATERIAL E MÉTODOS}

Foram realizados exames ecocardiográficos em modos B, M e Doppler em 27 indivíduos da espécie Leopardus tigrinus, mantidos em cativeiro na Fundação Parque Zoológico de São Paulo, alocados em jaulas coletivas. Todos os animais estudados eram adultos, sendo 59,3\% machos e $40,7 \%$ fêmeas.

Para a avaliação ecocardiográfica, os animais foram submetidos à contenção química com injeções intramusculares contendo xilazina e quetamina nas doses de 1 a $2 \mathrm{mg} / \mathrm{kg}$ e de $10 \mathrm{mg} / \mathrm{kg}$, respectivamente, com auxílio de jaula de contenção.

Para a execução do exame ecocardiográfico, os animais foram mantidos tanto em decúbito lateral esquerdo quanto direito, conforme as janelas acústicas desejadas.

Os valores dos parâmetros ecocardiográficos obtidos neste estudo foram submetidos à análise estatística descritiva, para o cálculo do valor médio e do desvio-padrão. A diferença entre as médias foi significativa quando $\mathrm{P}<0,05$.

\section{RESULTADOS}

Na Tab. 1 são apresentados a média, o desviopadrão e a variação dos diferentes parâmetros ecocardiográficos estudados.

A parede do ventrículo direito não pôde ser claramente delimitada nos exames ecocardiográficos da espécie estudada, impossibilitando mensuração acurada das dimensões internas dessa cavidade cardíaca.

Dez em 27 (37\%) dos animais estudados apresentaram alterações valvares, sendo que em sete indivíduos diagnosticou-se insuficiência da valva mitral (Fig. 1 e 2); dois apresentaram escape da referida valva e apenas um, insuficiência de ambas as valvas atrioventriculares, mitral e tricúspide.

\section{DISCUSSÃO}

É de suma importância ressaltar a falta de literatura no que se refere aos parâmetros ecocardiográficos de felinos selvagens. Os trabalhos encontrados referiam-se sempre ao gato doméstico, sendo que poucos deles apresentam informações sobre valores de referência que, quando existentes, abrangem apenas alguns dos parâmetros e/ou não apresentam o mesmo protocolo anestésico utilizado neste estudo.

A contenção química com uso de anestésicos é utilizada em gatos para facilitar a realização de exame ecocardiográfico detalhado; entretanto, essa conduta pode promover alterações no funcionamento do sistema cardiovascular, influenciando diretamente as mensurações obtidas no exame (Fox et al., 1985; Jacobs e Knight, 1985), tanto é que o ecocardiograma tem sido utilizado para acessar os efeitos de agentes químicos na função ventricular (Allen e Downey, 1983). 
Avaliação de alguns parâmetros...

Tabela 1. Parâmetros ecocardiográficos obtidos em gatos-do-mato (Leopardus tigrinus), anestesiados com quetamina e xilazina

\begin{tabular}{|c|c|c|c|c|}
\hline Parâmetro & Média & Desvio-padrão & Variação & Número \\
\hline SIVd (cm) & 0,38 & 0,06 & $0,25-0,50$ & 26 \\
\hline SIVs (cm) & 0,54 & 0,07 & $0,39-0,67$ & 26 \\
\hline DIVEd $(\mathrm{cm})$ & 1,69 & 0,19 & $1,30-2,08$ & 26 \\
\hline DIVEs (cm) & 1,19 & 0,20 & $0,81-1,64$ & 26 \\
\hline PLVEd $(\mathrm{cm})$ & 0,39 & 0,05 & $0,29-0,52$ & 26 \\
\hline PLVEs (cm) & 0,59 & 0,09 & $0,42-0,75$ & 26 \\
\hline $\mathrm{FC}(\mathrm{bpm})$ & 92,46 & 20,43 & $60,0-130,0$ & 24 \\
\hline $\mathrm{AE}(\mathrm{cm})$ & 0,92 & 0,15 & $0,62-1,27$ & 26 \\
\hline Ao $(\mathrm{cm})$ & 0,88 & 0,11 & $0,62-1,09$ & 26 \\
\hline $\mathrm{AE} / \mathrm{Ao}$ & 1,04 & 0,08 & $0,96-1,11$ & 26 \\
\hline $\mathrm{DC}$ & 0,44 & 0,16 & $0,10-0,80$ & 24 \\
\hline FE\% $\%(\%)$ & 60,19 & 11,41 & $27,0-74,0$ & 27 \\
\hline VEJ (ml) & 4,94 & 1,55 & $1,66-8,32$ & 27 \\
\hline VOLs (ml) & 3,38 & 1,58 & $1,16-7,64$ & 27 \\
\hline $\operatorname{VOLd}(\mathrm{ml})$ & 8,32 & 2,57 & $4,16-14,10$ & 27 \\
\hline FS\%SIV (\%) & 46,24 & 21,23 & $22,0-116,0$ & 27 \\
\hline FS\% (\%) & 30,10 & 7,07 & $11,3-39,7$ & 27 \\
\hline FS\%PLVE (\%) & 53,94 & 21,33 & $28,2-102,7$ & 27 \\
\hline E-S (cm) & 0,27 & 0,08 & $0,17-0,41$ & 21 \\
\hline Vao $(\mathrm{cm} / \mathrm{seg})$ & 56,31 & 13,01 & $28,8-77,4$ & 14 \\
\hline Mit E (cm/seg) & 42,29 & 13,30 & $23,8-56,7$ & 11 \\
\hline Mit A (cm/seg) & 35,37 & 11,42 & $22,0-55,2$ & 11 \\
\hline VAP (cm/seg) & 50,23 & 13,48 & $32,2-66,9$ & 7 \\
\hline Peso $(\mathrm{kg})$ & 2,344 & 0,31 & $1,82-2,89$ & 27 \\
\hline
\end{tabular}

SIVd: espessura do septo interventricular na diástole; SIVs: espessura do septo interventricular na sístole; DIVEd: diâmetro do ventrículo esquerdo na diástole; DIVEs: diâmetro do ventrículo esquerdo na sístole; PLVEd: espessura da parede livre do ventrículo esquerdo na diástole; PLVEs: espessura da parede livre do ventrículo esquerdo na sístole; FC: freqüência cardíaca; AE: diâmetro do átrio esquerdo; Ao: raiz da artéria aorta; $\mathrm{AE} / \mathrm{Ao}$ : relação entre $\mathrm{AE}$ e Ao; DC: débito cardíaco; FE\%: fração de ejeção; VEJ: volume de ejeção; VOLs: volume na sístole; VOLd: volume na diástole; FS\% SIV: fração de encurtamento do septo interventricula; FS\%: fração de encurtamento; FS\% PLVE: fração de encurtamento da parede livre do ventrículo esquerdo; E-S: distância entre o ponto E da valva mitral e o SIV; VAo: velocidade do fluxo sistólico sangüíneo na artéria aorta; Mit E: velocidade de enchimento ventricular rápido; Mit A: velocidade de enchimento átrio-ventricular; VAP: velocidade do fluxo sistólico sangüíneo na artéria pulmonar.

A quetamina é um agente anestésico dissociativo (Cruz et al., 2000; Valadão, 2002; Carvalho et. al., 2006), que estimula, indiretamente, o sistema cardiovascular, sendo, também, inotrópico positivo (Valadão, 2002), além de causar aumento da pressão arterial, da freqüência e do débito cardíacos (Fox et al., 1985; Jacobs e Knight, 1985; Valadão, 2002, Carvalho et al., 2006).
A quetamina nunca deve ser utilizada como único anestésico em cães e gatos, necessitando de associação com agente sedativo ou tranqüilizante para prevenir efeitos colaterais como excitação, aumento de tônus vascular, hipertensão e salivação (Cruz et al., 2000; Cortopassi e Fantoni, 2002). 


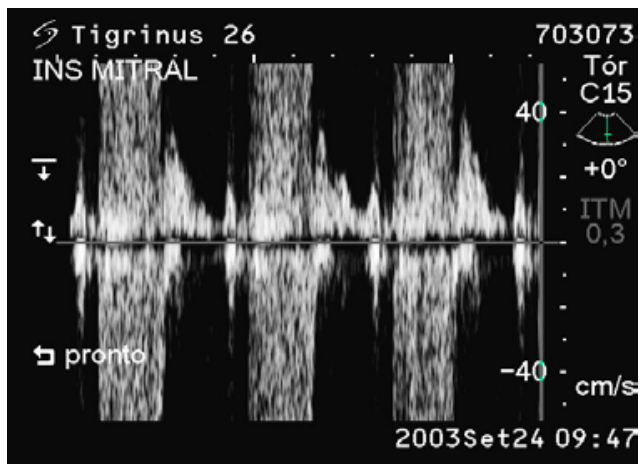

Figura 1. Imagem de insuficiência de valva mitral com uso de Doppler pulsátil, de um animal da espécie Leopardus tigrinus.

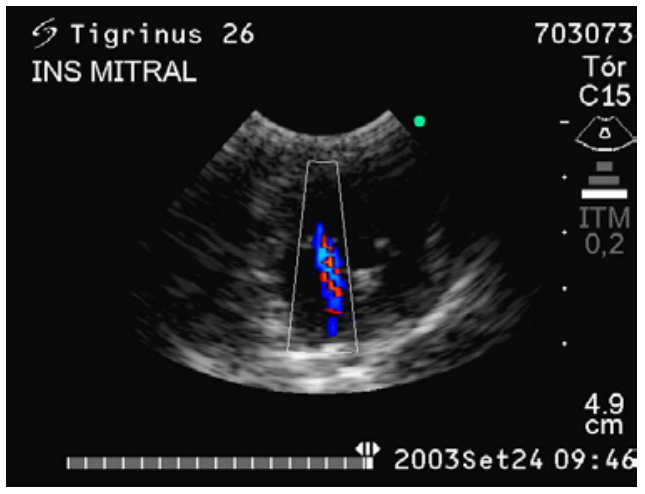

Figura 2. Imagem de insuficiência da valva mitral com uso de Doppler colorido, de um animal da espécie Leopardus tigrinus.

Um dos agentes que podem ser associados à quetamina, com sucesso, é a xilazina, fármaco $\alpha$ 2 agonista, que, no sistema cardiovascular, inicialmente leva a um aumento de pressão arterial seguido de hipotensão, diminui a freqüência e o débito cardíacos (Allen e Downey, 1983; Haskins e Patz; Farver, 1986; Cortopassi e Fantoni, 2002).

A xilazina e a quetamina são agentes anestésicos com efeitos cardiovasculares opostos, entretanto a depressão causada pela xilazina não é contrabalanceada pela ação simpatomimética da quetamina, sendo assim, essa associação leva à bradicardia e à hipotensão (Hsu e Lu, 1984; Cortopassi e Fantoni, 2002).

Desta maneira, os parâmetros ecocardiográficos estatisticamente semelhantes entre os animais do presente estudo (Leopardus tigrinus) e gatos domésticos anestesiados somente com quetamina (Fox et al., 1985) são poucos, pois os efeitos estimulantes da quetamina sobre o sistema cardiovascular são dominados pela ação depressora da xilazina. Tais parâmetros foram: SIVd $(p=0,295)$ e de AE/Ao $(p=0,116)$.

$\mathrm{O}$ único parâmetro comum entre Leopardus tigrinus e gatos domésticos anestesiados com xilazina, estudados por Allen e Downey (1983) foi a fração de encurtamento da fibra miocárdica $(\mathrm{p}=0,71)$. Índice este que demonstra a ação depressora simpatolítica, provocada por esse agente. A xilazina tem como efeito deprimir a dimensão diastólica do ventrículo esquerdo, que se relaciona à pré-carga e à porcentagem de mudança do diâmetro e à velocidade de encurtamento da fibra, que são índices relativos à contratilidade miocárdica (Allen e Doney, 1983).

Como observado em trabalhos anteriores (Fox et al., 1985; Jacobs e Knight, 1985; Carvalho et al., 2006), a anestesia leva a alterações na função cardíaca, o que reflete diretamente sobre os parâmetros ecocardiográficos. Portanto, pouca relação existe entre gatos domésticos não anestesiados, estudados por Jacobs e Knight (1985) e por Sisson et al. (1991), e os animais da espécie estudada, que sofreram contenção química com a associação de xilazina e quetamina.

Diferentemente do que ocorre no gato doméstico, em que as afecções do miocárdio, principalmente a $\mathrm{CMH}$, constituem-se nas cardiopatias de maior ocorrência (Moise et al., 1986; Rush, 1998; Rishniw, 2000; Häggström, 2002; Ferasin et al., 2003), grande parte dos felídeos selvagens estudados apresentaram alterações valvares, principalmente de valva mitral. No gato doméstico, a insuficiência da valva mitral é freqüentemente diagnosticada em associação com os casos de $\mathrm{CMH}$, associação essa não detectada na espécie estudada.

A displasia de valvas atrioventriculares é a cardiopatia congênita mais freqüentemente observada em gatos domésticos (Bonagura e Herring, 1985; Bonagura, 2000; Sisson et al., 2004). Já a insuficiência adquirida de valvas mitral ou tricúspide apresenta prevalência presumidamente baixa em gatos domésticos, sem doença miocárdica primária (Bonagura, 2000; Kvart e Häggström, 2004). Como a idade exata 
dos animais deste estudo é desconhecida, não se pode afirmar se a alta prevalência de alterações valvares nesses felídeos selvagens ocorre por doença congênita ou adquirida.

\section{CONCLUSÕES}

O uso de anestésicos altera a função cardiovascular; a xilazina apresenta maiores efeitos no sistema cardiovascular quando em associação com a quetamina; a diminuição da fração de encurtamento da fibra miocárdica se deve ao efeito depressor da xilazina; há pouca semelhança entre os parâmetros ecocardiográficos de gatos domésticos sem contenção química e de gatos-do-mato anestesiados com quetamina e xilazina.

\section{REFERÊNCIAS BIBLIOGRÁFICAS}

ALLEN, D.G.; DOWNEY, R.S. Echocardiographic assessment of cats anesthetized with xylazine-sodium pentobarbital. Can. J. Comp. Med. Vet. Sci., v.47, p.281-283, 1983 .

BONAGURA, J. D. Feline echocardiography. $J$. Feline Med. Surg., v.2, p.147-151, 2000.

BONAGURA, J.D.; HERRING, D.S. Echocardiography. Congenital heart disease. Vet. Clin. N. Am.: Small Anim. Pract., v.15, p.1195-1208, 1985.

CARVALHO, R.O.; ARAÚJO, R.B.; SILVA, E.F. Ecocardiografia modo Dopler pulsado em gatos clinicamente sadios. Arq. Bras. Med. Vet. Zootec., v.58, p.333-340, 2006.

CORTOPASSI, S.R.G.; FANTONI, D.T. Medicação pré-anestésica. In: FANTONI, D.T.; CORTOPASSI, S.R.G. Anestesia em cães e gatos. São Paulo: Roca, 2002. p.151-158.

CRUZ, M.L.; LUNA, S.P.L.; CASTRO, G.B. et al. A preliminar trial comparison of several anesthetic techniques in cats. Can. Vet. J., v.41, p.481-485, 2000.

FERASIN, L.; STURGESS, C.P.; CANNON, M.J. et al. Feline idiopathic cardiomyopathy: a retrospective study of 106 cats (1994-2001). J. Feline Med. Surg., v.5, p.151-159, 2003.

FOX, P.R.; BOND, B.R.; PETERSON, M.E. Echocardiographic values en healthy cats sedated with ketamine hydrochloride. Am. J. Vet. Res., v.46, p.1479-1484, 1985.
HAGGSTROM, J. Hypertrophic cardiomyopathy in cats - it used to be so simple! J. Feline Med. Surg., v.5, p.139-141, 2003.

HASKINS, S.C.; PATZ, J.D.; FARVER, T.B. Xylazine and xylazine-ketamine in dogs. Am. J. Vet. Res., v. 47, p. 636-641, 1986.

HSU, W.H.; LU, Z. Effect os yohimbine on xylazineketamine anesthesia in cats. J. Am. Vet. Med. Assoc., v.185, p.886-888, 1984.

JACOBS, G.; KNIGHT, D.H. Change in M-mode echocardiographic values in cats given ketamine. $\mathrm{Am}$. J. Vet. Res., v.46, p.1712-1713, 1985.

KVART, C.; HÄGGSTRÖM, J. Cardiopatia valvar adquirida. In: ETTINGER, S. J.; FELDMAN, E. C. Tratado de medicina interna veterinária: doenças do cão e do gato. 5. ed. Rio de Janeiro: Guanabara Koogan, 2004. v.1. p.833-846.

MOISE, N.S.; DIETZE, A.E.; MEZZA, L.E. et al. Echocardiography, electrocardiography, and radiography of cats with dilatation cardiomyopathy, hipertrophic cardiomyopathy, and hyperthyroidism. Am. J. Vet. Res., v.47, p.1477-1486, 1986.

RISHNIW, M. Radiography of feline cardiac disease Vet. Clin. N. Am.: Small Anim. Pract., v.30, p.395$425,2000$.

RUSH, J. E. Therapy of feline hypertrophic cardiomyopathy. Vet. Clin. N. Am.: Small Anim. Pract., v.28, p.1459-1479, 1998.

SCHWARTZ, D. S. Cardiomiopatia hipertrófica. In: SOUZA, H. J. M. Coletâneas em medicina e cirurgia felina. Rio de Janeiro: L. F. Livros, 2003. p.25-41.

SISSON, D. D.; KNIGHT, D. H.; HELINSKI, C. et al. Plasma taurine concentration and M-mode echocardiographic measures in healthy cats and cats with dilated cardiomyopathy. J. Vet. Int. Med., v.5, p.232-238, 1991.

SISSON, D.D.; THOMAS, W.P.; BONAGURA, J.D. Cardiopatia congênita. In: ETTINGER, S. J.; FELDMAN, E. C. Tratado de medicina interna veterinária: doenças do cão e do gato. 5 . ed. Rio de Janeiro: Guanabara Koogan, 2004. v.1. p.781-832.

VALADÃO, C. A. A. Anestésicos dissociativos. In: FANTONI, D. T.; CORTOPASSI, S. R. G. Anestesia em cães e gatos. São Paulo: Roca, 2002. p.165-173.

YAMATO, R. J. Estudo dos parâmetros ecocardiográficos em modo $M$ de cães da raça poodle miniatura, clinicamente sadios. 2001. 94f. Dissertação (Mestrado em Clínica Veterinária) - Faculdade de Medicina Veterinária e Zootecnia, Universidade de São Paulo, São Paulo. 